\title{
POBLACIONES Y COMUNIDADES DE ALGAS BENTONICAS EN LA COSTA CATALANA
}

\author{
L. POLO ALBERTI* y J. SEOANE - CAMBA**
}

\section{RESUMEN:}

Las poblaciones de algas bentónicas forman comunidades que en unos lugares están poco diferenciadas y en otros constituyen comunidades definidas de un cierto valor in dicativo. En este trabajo se comentan diversos perfiles de la costa catalana, confeccionadas según esquemas tomados sobre el terreno y auxiliados por fotografías submarinas.

Los perfiles, tomados en varias localidades de la costa, revelan algunos horizontes y facies característicos del Mediterráneo occidental, lo que nos permite a modode síntesis, tabular según su exposición al oleaje y a la luz, las facies más conspicuas y mejor caracterizadas.

\section{SUMMARY:}

The populations of benthic algae form communities which are little differenciated in some spots while in others they constitute definite communities with some indicative value. Several profiles of the catalan coast are treated in this paper they have been drawn in accordance with outlines taken on the ground and with the aid of submarine pictures.

The profiles, taken at several spots of the coast, reveal some horizonts and facies which are characteristic of the west Mediterranean, this allows us to tabulate the facies more evidents and better characterized according to their exposure to the swell and to the light.

El estudio sistemático y corológico de las algas bentónicas de la costa catalana ha sido dado a conocer en trabajos anteriores por nosotros mismos (Seoane-Camba \& Polo, 1974 y 1977; Seoane-Camba, 1975; Polo \& Seoane-Camba, 1976 a y b) y pretendemos exponer en la presente comunicación los principales grupos de poblaciones y comunidades que con cierta regularidad aparecen en dicha costa.

Existen varios procedimientos para describir bionómicamente las poblaciones y comunidades de los seres vivos; una de ellas consiste en el establecimiento de unidades sintaxonómicas que aparecen regularmente cuando las condiciones de sustrato, humedad, luz y otros factores alcanzan determinadas cotas y que seme-

(*) Departamento de Biología. Colegio Universitario de Gerona.

(**) Catedra de Botánica. Facultad de Farmacia. Universidad de Barcelona.

comunicación presentada al III Simposio Nacional de Botánica Criptogámica. Málaga, 1978. 
jan, en cierta medida, las técnicas fitosociológicas utilizadas corrientemente en la vegetación terrestre.

En la descripción de las poblaciones y comunidades que nos ocupan, carecemos de dichas unidades básicas que puedan utilizarse para expresar claramente la realidad fisionómica de la costa catalana. Por otra parte intentamos evitar en lo posible la descripción de comunidades que puedan ser muylocalistas y por lo tanto de escaso interés a nivel general.

Las algas marinas bentónicas podemos considerarlas como organismos intermedios entre la vegetación terrestre y los organismos inferiores; la estabilidad de sus comunidades es mucho menor en general, que la que se da en los vegetales terrestres y mayor que la que se da en los organismos unicelulares; las variaciones en su distribución y el efecto que las pequeñas fluctuaciones en los factores ecológicos ejerce sobre sus comunidades y su biología en general, son muy grandes, por lo que consideramos que la descripción y el establecimiento de comunidades tipo (p. ej. asociaciones) sin haber sido contrastadas estadísticamente a partir de un gran número de datos de observación, puede conducirnos a un número infinito de tales unidades y por lotanto puede ser en la práctica contraproducente para la comprensión real de estos hechos. Desde este punto de vista damos a conocer mediante esquemas y descripciones (tomados sobre el terreno y auxiliados por fotografías submarinas), la distribución de las principales poblaciones que hemos encontrado en la costa catalana.

La localidades estudiadas que hemos tomado como tipo, se extienden desde el macizo de Cabo Begur hasta los salientes calcáreos de Vilanova i Geltrú y corresponden a distintos puntos de Sa Tuna, Sant Feliu de Guíxols, Blanes, Garraf y Vilanova i Geltrú, localidades que se hallan representadas en la figura 1. En cuanto a las características geológicas de esta costa, determinantes en la elección de las localidades de estudio, las expusimos y comentamos en nuestros anteriores trabajos (Equipo de bentos, 1974; Polo \& Seoane-Camba, 1976 a; Polo 1978).

Exponemos a continuación los esquemas y descripciones de las localidades citadas. En los esquemas las especies se representan de forma convencional, siguiendo en gran parte la simbología utilizada por Lewis (1964), Bellan-Santini (1969) y Niell (1976) además de otros símbolos propios pues varias especies no se hallan representadas en estas obras. La clave de los símbolos se encuentra en la figura 2; varias especies pertenecientes a un mismo género tienen a menudo una representación única, la especie concreta (si se ha determinado) se indica en el texto. Solo se representan las especies más abundantes, las acompañantes y epífitas se citan en los comentarios. Los organismos animales no se han representado; cuando son abundantes y forman poblaciones densas nos referimos a ellos por su nombre genérico.

\section{Sa Tuna}

1.-El lugar estudiado corresponde a Illa Negra (esquematizado en la figura 3A), se trata de un lugar batido donde aparecen muy claros los horizontes de Rissoe- 


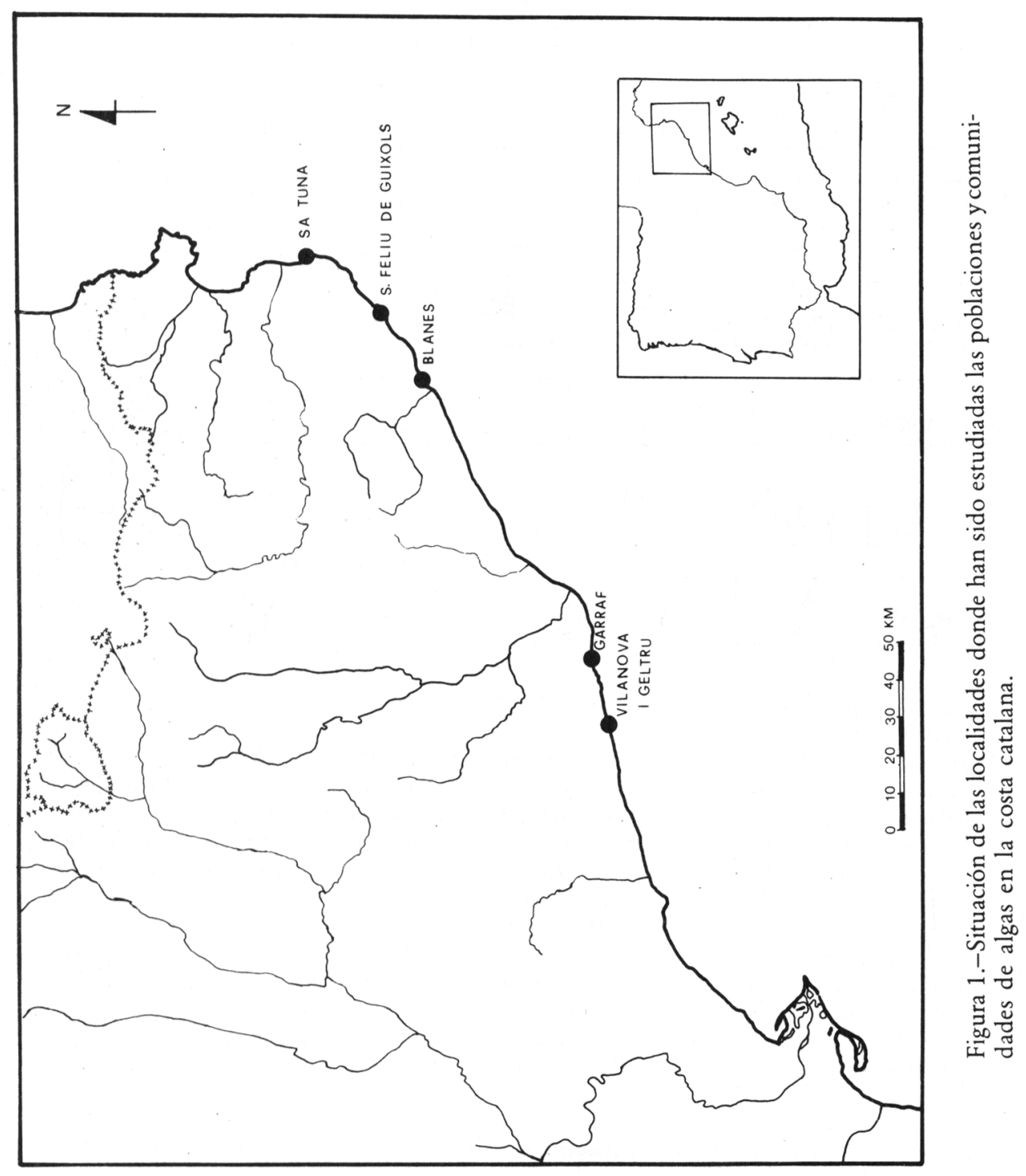



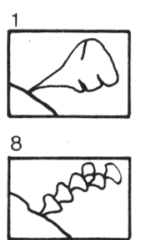

15

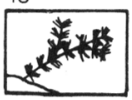

22

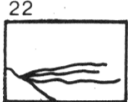

29

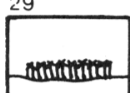

36

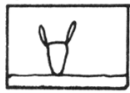

43

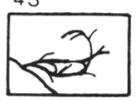

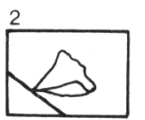

9

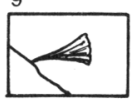

16

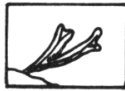

23

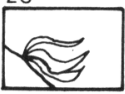

30

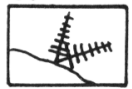

37

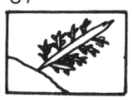

44

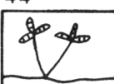

3

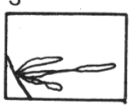

10

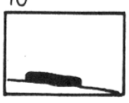

17

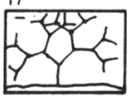

24

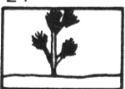

31

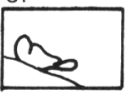

38

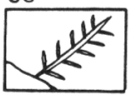

45

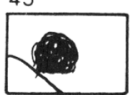

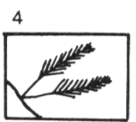

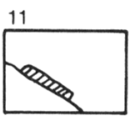

18
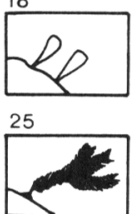

32

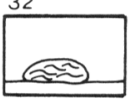

39

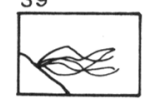

46

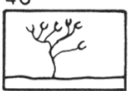

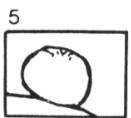
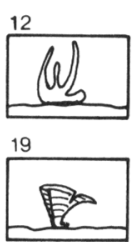

26

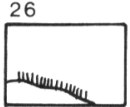

33

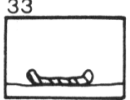

40

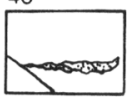

47

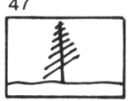

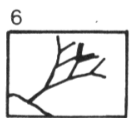

13
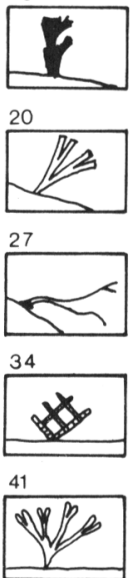

48

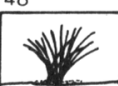

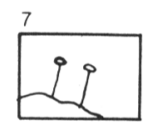

14
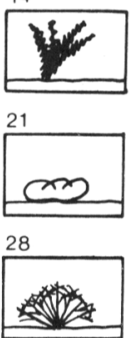

35

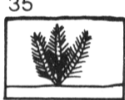

42

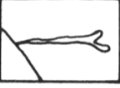

Figura 2.-Representación convencional de las especies que aparecen en los esquemas de las figuras 3,4 y 5 .

Algas: 1. Ulva lactuca, 2. Ulva rigida, 3. Enteromorpha, 4. Bryopsis, 5. Codium bursa, 6. Codium tomentosum, 7. Acetabularia mediterranea, 8. Halimeda tuna, 9. Udotea petiolata, 10. Nemoderma tingitana, 11. Ralfsia verrucosa, 12. Mesogloia vermiculata, 13. Halopteris scoparia, 14. Halopteris filicina, 15. Cladostephus verticillatus, 16. Dictyopteris membranacea, 17. Dictyota dichotoma, 18. Dilophus fasciola, 19. Padina pavonia, 20. Taonia atomaria, 21. Colpomenia sinuosa, 22. Scytosiphon lomentaria, 23. Petalonia fascia, 24. Cystoseira caespitosa, 25. Cystoseira, 26. Bangia fuscopurpurea, 27. Nemalion belminthoides, 28. Liagora viscida, 29. Gelidium, 30. Pterocladia capillacea, 31. Peyssonnelia, 32. Lithophyllum tortuosum, 33. Coralináceas incrustantes, 34. Amphiroa rigida, 35. Corallina mediterranea, 36. Jania rubens, 37. Halymenia floresia, 38. Grateloupia filicina, 39. Gracilaria, 40. Rissoella verruculosa, 41. Gymnogongrus norvegicus, 42. Petroglosum nicaeense, 43. Gigartina acicularis, 44. Gastroclonium clavatum, 45. Falkenbergia rufolanosa, 46. Ceramium, 47. Laurencia. Cormófitos: 48. Posidonia oceanica.

Ila verruculosa, Lithophyllum tortuosum y de Cystoseira mediterranea. A unos 2-3 m., de profundidad donde la acción de las olas está amortiguada, las rocas que descienden en suave pendiente se hallan ocupadas por Padina pavonia y Liagora viscida, los lugares de pendiente más acentuada presentan poblaciones de Halopteris filicina y Dictyota dichotoma. Estos dos horizontes infralitorales aparecen, evidentemente, mezclados o superpuestos en muchos puntos.

A partir de los 5-6 m., de profundidad las especies más abundantes son $\mathrm{Co}^{-}$ dium bursa y Codium tomentosum. Otras especies de la zona infralitoral superior, no representadas pero también abundantes son: Asparagopsis armata, Laurencia obtusa, Sphaerococcus coronopifolius, también y en grietas a unos 3-4 m., de profundidad Peyssonnelia squamaria, Halimeda tuna y Udotea petiolata. 


\section{Sant Feliu de Guíxols.}

2.-En Punta Prima tomamos como ejemplo un lugar poco batido hasta unos 5-6 m., de la profundidad como el representado en la figura 3-B.

Nivel litoral ocupado por cespedines de Gelidium sp. con Laurencia obtusa y Falkenbergia rufolanosa inmediatamente por debajo predominan Padina pavonia, Halopteris scoparia y Dictyota dichotoma (se trata por lo general de D. dichotomaf. implexa).

Al descender las rocas en pendiente suave hacia el fondo, son abundantes Laurencia obtusa, Colpomenia sinuosa y Dictyota dichotoma a compañadas por coralináceas incrustantes, también Padina pavonia y numerosas pequeñas masas de Amphiroa rigida y otras especies que no se han representado como Corallina mediterranea, Cystoseira stricta y Jania longifurca.

A partir de unos 3 metros de profundidad y hasta cerca del fondo arenoso (5-6 m.) abundan los feófitos: Cladostephus verticillatus, Halopteris filicina y Dictyota dichotoma, formando las dos primeras masas considerables. Ya cerca del fondo predominan los clorófitos: Codium bursa y Codium tomentosum. Clorófitos y feófitos se superponen ampliamente, nosotros indicamos básicamente el predominio de las poblaciones.

En Pta. Prima son frecuentes los fondos arenosos con rocas que sobresalen un par de metros de la arena, como la representada en la misma figura, con Halymenia floresia y Peyssonnelia rubra en la parte menos iluminada; en estos lugares es abundante Microcosmus recubierto de algas: Peyssonnelia rubra como más abundante, también Botryocladia boergeseni, Gelidium latifolium y otras especies). La pared vertical de la roca orientada al oeste se encuentra poblada por Halimeda tuna y Udotea petiolata, si bien los ejemplares no alcanzan el desarrollo que presentan a mayor profundidad. La parte superior es semejante a las rocas de la costa con Cladostephus verticillatus, Halopteris filicina, Dictyota dichotoma, además de coralináceas incrustantes y Amphiroa rigida; en la parte orientada a levante y cerca del fondo predominan Codium bursa y Codium tomentosum.

3.-Las hendiduras y cubetas estrechas son frecuentes en Pta. Prima como la de la figura 3-D, donde representamos una hendidura que se ensancha en su parte central y cuya profundidad es de 1,2 a 1,5 metros, con renovación del agua contínua y agitación escasa. El esquema corresponde a un corte transversal de forma que la cara orientada al sur se hallaría en la boca de la hendidura y la cara orientada al norte en su parte media.

Siguiendo el esquema de izquierda a derecha, las especies más abundantes son: Nemalion belminthoides un poco por encima del nivel del agua y en forma de ejemplares dispersos, la zona litoral ocupada por una población densa de Pterocladia capillacea, a continuación y casi hasta el fondo Laurencia obtusa, Corallina mediterránea, Dictyota dichotoma y Halopteris scoparia. En la parte más iluminada del fondo, Cystoseira caespitosa y Padina pavonia con Taonia atomaria menos abundante.

En la cara orientada al norte las poblaciones son sustacialmente distintas: Peyssonnelia sp. y Udotea petiolata en el fondo, sustituyéndose gradualmente por 


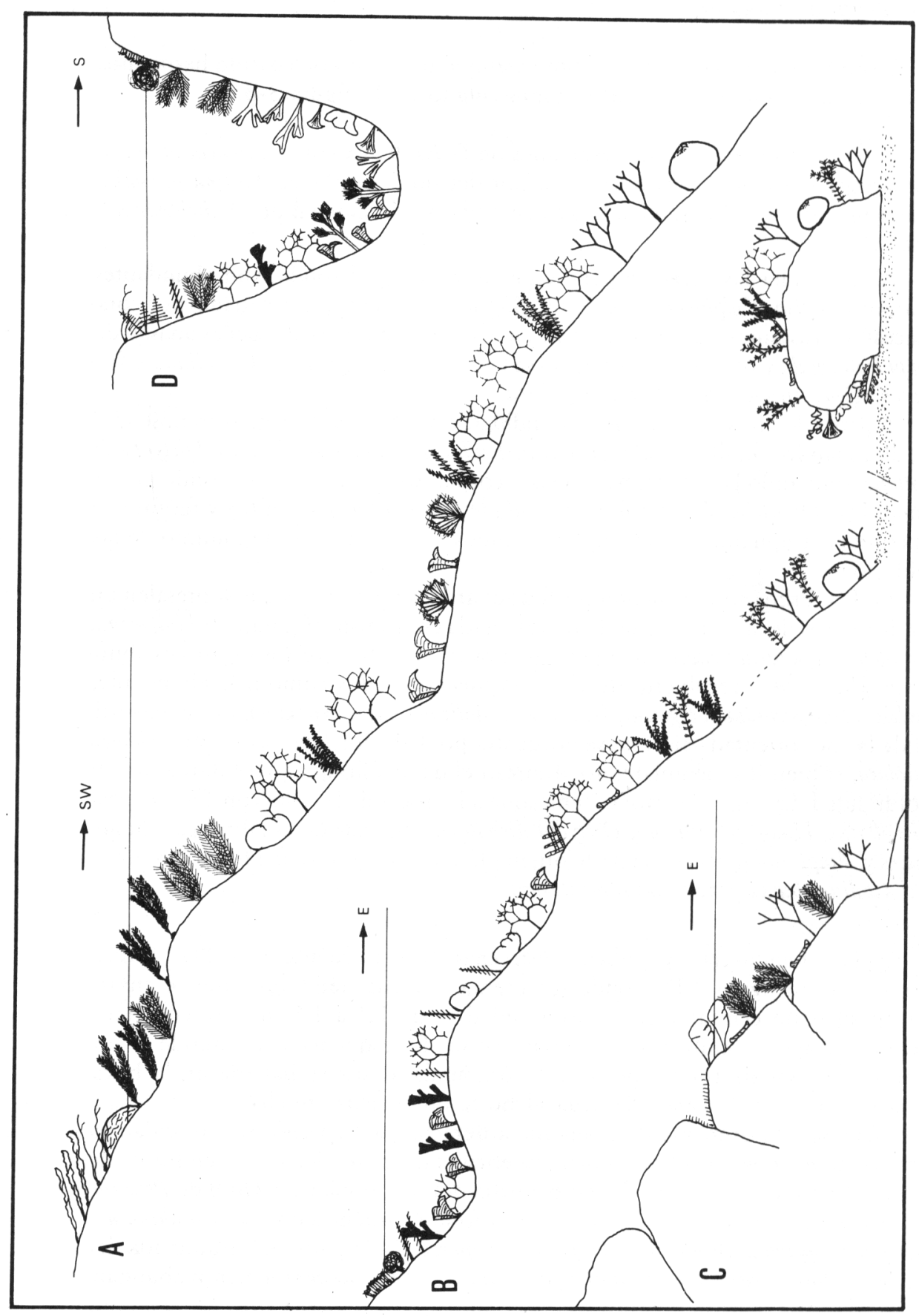

ะ. 过芒 更 हᄐำ क्ष 뇽ำ $\infty$. 的娄官

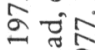
눙 8 范 잉을 ธี 율 छํํㅇ

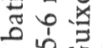

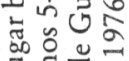
$\exists$ 워

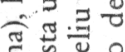
焉江。

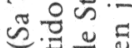

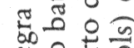
ž 吾证证

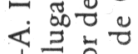
i. 뜹.

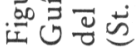


Dictyopteris membranacea y Taonia atomaria hasta unos 50-40 cm., de la superficie entre una población cada vez más densa de Corallina mediterranea (con epífitos: Falkenbergia rufolanosa y Acrosorium reptans) que llegan hasta cerca del nivel $0 \mathrm{~m}$., donde predominan Gelidum sp. y Falkenbergia rufolanosa.

4.-Las cubetas anchas y poco profundas, por tanto bien iluminadas presentan su fondo ocupado básicamente por Padina pavonia y Cystoseira caespitosa en los lugares de agitación escasa o moderada.

Tomamos como ejemplo una cubeta en Sant Pol, ancha de 4 a 5 metros y poco profunda $(40-60 \mathrm{~cm}$.) que está en comunicación con mar abierto porsu parte norte con rocas que llegan casi hasta la superficie, de forma que las aguas de la cubeta sufren poca agitación a pesar de hallarse en un saliente batido.

Siguiendo el esquema de la figura 4-B de izquierda a derecha, se halla Cystoseira fimbriata en ejemplares dispersos y algo distintos de los de lugares batidos (forma rosetas de unos $15 \mathrm{~cm}$., de diámetro en verano). El fondo de la cubeta se halla ocupado por Padina pavonia, Cystoseira caespitosa, Halopteris scoparia y coralináceas incrustantes. En la orientación en que tomamos el esquema, las rocas de unos 2 metros de altura, separan la cubeta del mar abierto y son rebasadas únicamente con mar bastante movida; estas rocas se hallan casi totalmente recubiertas de Chtamalus.

En las rocas de mar abierto, horizontes de Rissoella verruculosa y Lithophyllum tortuosum en la zona supralitoral; esta especie marca precisamente el nivel 0 metros en su parte inferior. Cystoseira mediterranea a continuación Dilophus fasciola, Laurencia papillosa y hasta $1 \mathrm{~m}$., de profundidad, Corallina mediterranea y Dictyota dichotoma.

5.-En este caso el ejemplo corresponde a El Molar, tomado en lugares poco batidos y con rocas que llegan hasta cerca de la superficie, por lo que las poblaciones de algas son bastantes parecidas a las de ejemplos anteriores, con la particularidad de que en este lugar es abundante Acetabularia mediterranea.

En el esquema correspondiente a la figura 4-A, el niel 0 m., se halla ocupado por Gelidium pusillum, Ulva lactuca y Ulva rigida con Corallina mediterranea, Jania corniculata, Padina pavonia, Halopteris scoparia, Dictyota dichotoma y Laurencia obtusa en la cubeta; Grateloupia filicina, Halopteris scoparia y Jania corniculata en rocas que llegan hasta cerca del nivel 0 metros. La hondonada que sigue a continuación ocupada por Liagora viscida junto a varias de las especies ya citadas. Las rocas descienden en pendiente y a partir de los 1,5-2 metros de profundidad están tapizadas por poblaciones densas de Cladostephus verticillatus y Acetabularia mediterranea, junto a ellas se presenta Dictyota dichotoma $f$. implexa que pasará a ser la especie más abundante a partir de los $4 \mathrm{~m}$., de profundidad.

A unos 5-6 m., el fondo rocoso es casi horizontal, acumulándose arena y sedimentos en las ondulaciones del terreno; estas circunstancias son las que permiten la instalación de Posidonia oceanica. Estas poblaciones de Posidonia en matas más o menos dispersas formando falsas praderas son frecuentes en la costa sobre rocas con sedimentos y arena. En El Molar acompañan a Posidonia oceanica: Codium bursa, Codium tomentosum y Acetabularia mediterranea. 


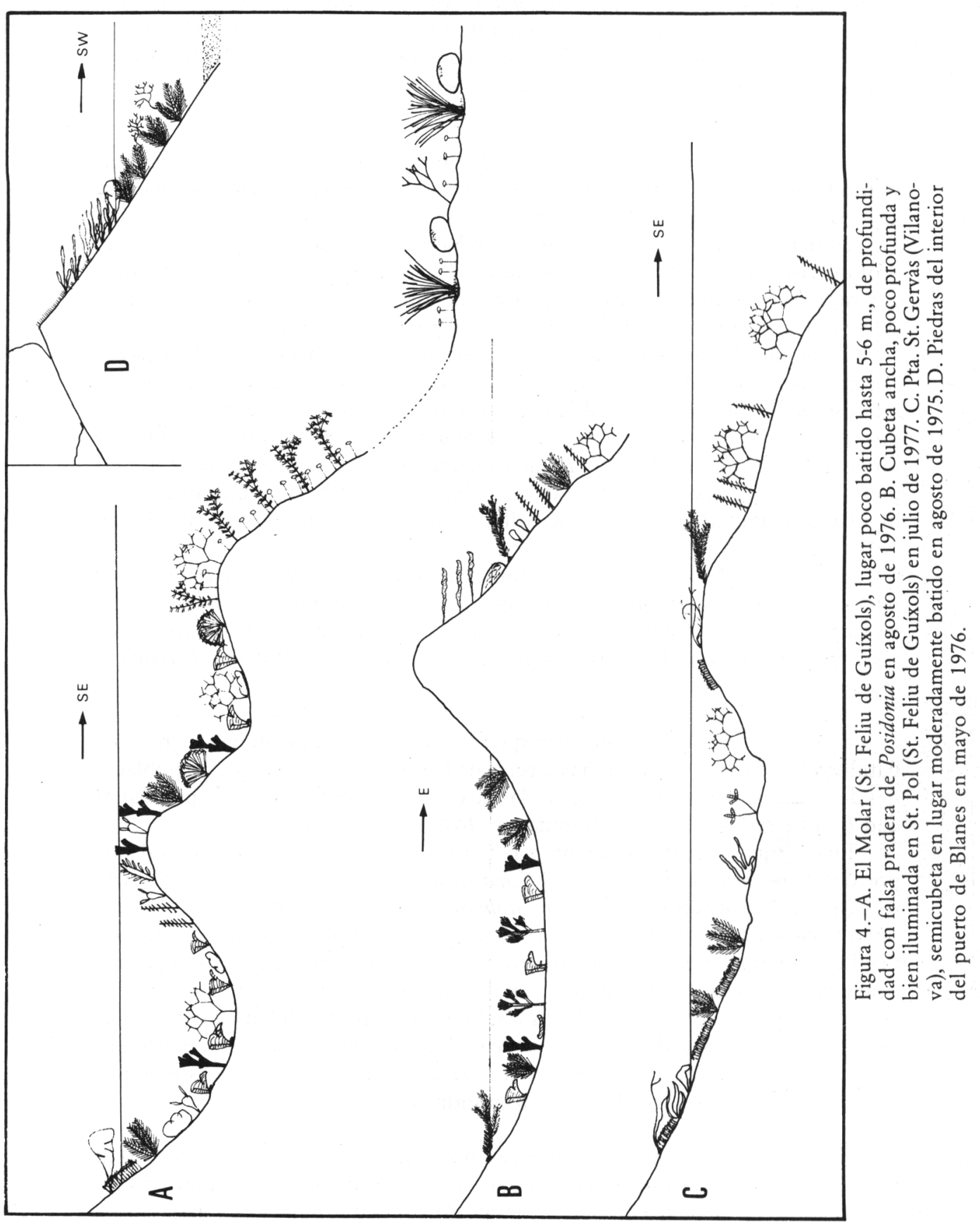


6.-El poblamiento algal del espigón del interior del puerto de Sant Feliu de Guíxols, resulta bastante distinto a los descritos hasta el momento en esta localidad.

En la figura 3-C, esquematizamos el espigón del interior del puerto, donde se unen las condiciones de aguas contaminadas con las de estancamiento. Zona supralitoral ocupada por masas densas y resbaladizas de Bagia fuscopurpurea; el nivel 0 m., con Ulva lactuca e inmediatamente por debajo, Corallina sp. (resulta difícil de clasificar como C. mediterranea o C. officinalis aunque por la situación podría tratarse de esta última) y coralináceas incrustantes: Lithophyllum incrustans y Pseudolitbophyllum orbiculatum entre otras. Codium tomentosum es frecuente y debido a las condiciones de escasa agitación llega hasta cerca del nivel litoral. En el espigón son muy abundantes las lapas (Patella).

\section{Blanes}

Las poblaciones de algas en esta localidad son bastante semejantes a las anteriores, las principales diferencias son manifiestan en los ejemplos que siguen a continuación.

7.-En la figura 5-D esquematizamos una hendidura existente en Pta. del Canò (Playa de Santa Cristina), cerrada en su parte sur y muy abierta al mar por el norte, en un lugar bastante batido al que se añade el rebote de las olas de una pared a otra de la hendidura. En el esquema cortamos la hendidura aproximadamente a 1 metro de su punto de cierre y donde el chapoteo es prácticamente máximo.

La zona supralitoral ocupada entre 1 y 1,5 m., de altura por manchas de Ralfsia verrucosa, luego a continuación y por debajo, horizonte de Rissoella verruculosa con Nemalion belminthoides menos abundante. Lithophyllum tortuosum forma "trottoir» bastante conspícuo y por debajo del mismo, ya en el nivel superior de la zona infralitoral, las rocas orientadas al oeste aparecen pobladas por Corallina mediterranea que forma un cesped denso, que alrededor de los 0,5 m., alterna con una población abundante de Petroglossum nicaeense.

Las rocas de la cara este se hallan ocupadas por Bryopsis sp., corallináceas incrustantes, Falkenbergia rufolanosa y Corallina mediterranea muy abundante formando cesped denso y poco alto entre el que aparecen pequeños ejemplares de Halimeda tuna.

8.-En Cala Forcanera se hallan poblaciones de algas en puntos moderadamente batidos, donde Rissoella verruculosa puede llegar a formar un horizonte ancho si las rocas descienden en pendiente moderada como en el ejemplo que esquematizamos en la figura 5-C. No hemos representado Lithopbyllum tortuosum ya que no llega a constituir «trottoir» sino que se halla formando pequeñas masas o en ejemplares dispersos; Gelidium sp. pl. forma cesped denso hasta cerca del nivel $0 \mathrm{~m}$. donde recubre el sustrato Nemoderma tingitana y por encima de ella Cystoseira crinita y/o Cystoseira mediterranea con numerosos epífitos: Chaetomorpha capillaris, Feldmannia paradoxa, Jania rubens y otras especies. 
A continuación y hasta 1,5 metros de profundidad predominan Corallina mediterranea, Jania rubens, Colpomenia sinuosa, Dictyota dichotoma, etc.

9.-Las piedras del interior del puerto de Blanes (frente a la lonja), se han representado en la figura 4-D, con las siguientes especies: Bangia fuscopurpurea formando masas densas en la zona supralitoral, que al aproximarse al nivel $0 \mathrm{~m}$., se van mezclando con Enteromorpha compressa y Scytosiphon lomentaria, hasta el nivel $0 \mathrm{~m}$., donde el dominio corresponde ya a Ulva lactuca.Por debajo y hasta el fondo de arena a unos $0,5 \mathrm{~m}$., de profundidad, cesped denso de Corallina mediterranea con masas abundantes de Ceramium sp.

\section{Garraf}

10.-La playa de Garraf presenta en algunas zonas, rocas planas a ras del agua, con gran cantidad de arena. Sobre estas rocas y sin que quede claro un orden o zonación (figura 5-A), se hallan las siguientes especies: Enteromorpha linza, E. ramulosa, E. compressa; Ulva lactuca y menos abundante Ulva rigida; Corallina mediterranea, Gelidium sp. y Gracilaria sp.

11.-Cubetas en lugares poco batidos en la parte sur de Garraf. La que representamos en la figura 5-B, comunica directamente con el mar por el suroeste, estando la parte de tierra poco iluminada ya que queda a la sombra de grandes bloques de piedra que no representamos en el esquema.

En estas condiciones un poco especiales hemos observado numerosos ejemplares de Gymnogongrus norvegicus en los alrededores del nivel $0 \mathrm{~m}$. Fondo de cubeta con Gastroclonium clavatum, coralináceas incrustantes, especialmente Lithophyllum incrustans; Corallina mediterranea y Corallina officinalis, esta última solo en algunos puntos; Gelidium crinale y Gelidium pulchellum alrededor de los $0 \mathrm{~m}$.

La roca que en el esquema separa la cubeta del mar, es sobrepasada más o menos por las olas y sostiene una población de Enteromorpha compressa en su parte superior, luego y cerca del nivel del agua Laurencia pinnatifida y Bryopsis sp. pl. formando matitas entre una población de Petalonia fascia, Enteromorpha y Ulva lactuca.

Por debajo del nivel litoral, cesped denso de Corallina mediterranea y Dilophus fasciola var. repens. Los mejillones (Mytilus) muy numerosos, van haciendose cada vez más abundantes en profundidad, predominando a partir de los $0,5 \mathrm{~m}$. Acompañan a estas especies Ceramium rubrum, Ceramium diaphanum, Hypoglossum woodwardi y otras que no detallamos.

12.-Debido a la especial naturaleza del sustrato, es característico en Garraf la formación de un voladizo o concavidad («sobreplomo") al pie de los acantilados, por encima del nivel donde rompen las olas y hasta $1,5 \circ 2 \mathrm{~m}$., de altura en lugares batidos o fuertemente batidos.

En estas circunstancias, incluso con mar poco movida, por el efecto de las olas y su rebote, las salpicaduras mojan facilmente toda la concavidad, lo que permite el asentamiento de algas hasta 1,5 m., o más de altura sobre el nivel litoral. En la par- 


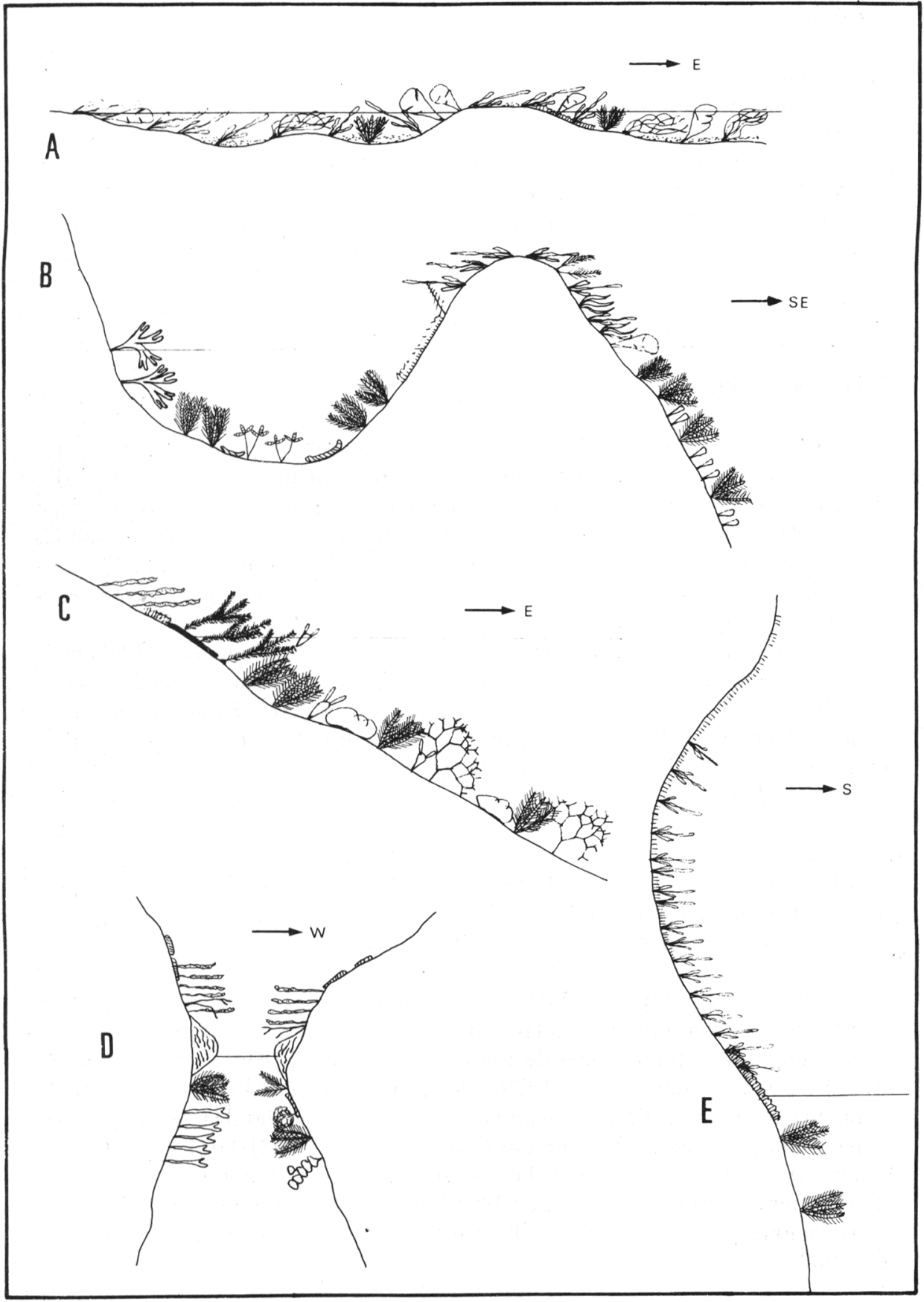

Figura 5.-A. Playa de Garraf, rocas planas enarenadas en junio de 1975. B. Rocas al sur de Garraf, cubeta semiiluminada en lugar poco batido en junio de 1975. C. Lugar moderadamente batido en cala Forcanera (Blanes) en mayo de 1976. D. Hendidura en lugar batido y poco iluminado en Pta. del Canò (Sta. Cristina, Blanes) en mayo de 1977. E. Concavidad al pie de los acantilados de Garraf en junio de 1975. 
te sur de Garraf las poblaciones de algas de este voladizo, tal como se esquematizan en la figura 5-E, son las siguientes: Bangia fuscopurpurea forma una masa contínua, densa, a plicada al sustrato y reteniendo abundante agua por capilaridad hasta aproximadamente 1,5 m., de altura y llegando a los $2 \mathrm{~m}$., en forma de mechones aislados. Enteromorpha compressa se presenta junto a Bangia en forma de ejemplares sueltos también aplicados contra el sustrato y forma población densa alrededor de los 0,5 metros al tiempo que Bangia desaparece progresivamente.

El nivel litoral está ocupado por Gelidium crinale formando masas cespitosas densamente apretadas y a continuación Corallina mediterranea congran cantidad de Mytilus.

\section{Vilanova i Geltrú}

13.-Son frecuentes en Pta Sant Gervàs las cubetas o semicubetas, pues a menudo las rocas que las separan del mar abierto únicamente rozan la superficie. En la figura 4-C representamos una semicubeta en un punto algo batido.

Nemalion belminthoides no muy abundante se halla en la zona supralitoral; $G e^{-}$ lidium pusillum forma cesped a continuación. Zona litoral ocupada por Petalonia fascia, Ulva lactuca y Ulva rigida, especies que en invierno, sin llegar a desaparecer, ceden la representación mayoritaria a Porphyra umbilicalis.

Cubeta ocupada en su primera mitad por Corallina mediterranea y Gelidium crinale. La segunda mitad, que en cierto modo queda algo resguardada de las olas, se caracteriza por la elevada densidad de anémonas de mar (cf. Anemonia sulcata) en las abundantes oquedades que allí presenta el sustrato; de forma más o menos dispersa las algas más abundantes son: Mesogloia vermiculata, Gastroclonium clavatum y Dictyota dichotoma.

La cresta de las rocas está ocupada por Gelidium crinale, Gigartina acicularis y Cystoseira mediterranea, a partir de aquí, empieza y hasta el fondo de arena a unos 2 m., de profundidad, una población densa de Mytilus con Laurencia pinnatifida y Dictyota dichotoma en matas más o menos dispersas.

Del estudio y comparación de las poblaciones de algas en estas localidades, se desprende, que son semejantes a las descritas por diversos autores en el Mediterráneo occidental: las poblaciones de algas de la costa de las Alberes por Feldmann (1937) y Boudouresque (1969 y 1970); el manual general de bentos para el Mediterráneo occidental de Peres \& Picart (1964); las descripciones de Córcega por Molinier (1960); la costa de Palermo por Giaccone \& Leo (1967) o de cabo Zafferano (Palermo) por Giaccone (1967); las cercanías de Nápoles por Feoli \& Bressan (1972). Tienen también muchas características en común con poblaciones más alejadas como las del mar de Alborán (Prieto, 1973) o del Adriático medio (Pignatti et al., 1967).

A modo de resumen, agrupamos las facies mejor caracterizadas y fácilmente visibles, según su exposición al oleaje y a la luz, siguiendo la división del sistema litoral propuesta por Seoane-Camba (1965 y 1969). 


\section{Lugares batidos}

Zona supralitoral, nivel inferior.

Horizonte de Rissoella verruculosa bien constituído en muchos puntos de la costa norte (Costa Brava, provincia de Gerona). No se ha detectado esta especie en el resto de la costa, posiblemente su ausencia sea atribuible al sustrato: vive sobre sustratos silíceos, más duros y resistentes a la abrasión marina que las rocas calcáreas que no permitirían, según suponemos, su asentamiento debido al rápido desmenuzamiento que sufren. Es en este sentido que atribuimos a Rissoella verruculosa su preferencia por sustratos silíceos.

Horizonte con Lithophyllum tortuosum, por debajo del anterior y con análoga distribución a lo largo de la costa, distribución que posiblemente sea atribuible a la misma causa.

Forma en los lugares batidos y fuertemente batidos el llamado "trottoir" por los franceses, cornisa de anchura variable y más o menos inclinada en su parte superior, que puede formar un saliente (de 10 a $20 \mathrm{~cm}$.) en paredes verticales de lugares muy batidos.

La amplitud de los horizontes de Rissoella verruculosa y Lithophyllum tortuosum depende además de la exposición a las olas, de la inclinación del sustrato (en pendientes moderadas los horizontes son más anchos) y de una buena iluminación.

Poblaciones de Bangia fuscopurpurea y Enteromorpha comprenssa, tal como se dan ẹn Garraf por las especiales condiciones que allí concurren. En esta forma, hasta más de 1,5 m., por encima del nivel litoral, no se ha observado en ninguna otra localidad.

\section{Zona litoral}

Horizonte de Cystoseira mediterranea bien constituído en muchos lugares de la costa norte, siendo menos abundante en la parte sur. Cystoseira mediterranea forma también poblaciones densas en rocas que apenas afloran al nivel del agua, acompañada por Mytilus en abundancia y a menudo por Cystoseira fimbriata.

En los lugares menos batidos se presenta un horizonte de Nemoderma tingitana y Cystoseira mediterranea y/o Cystoseira crinita, como en cala Forcanera de Blanes.

Tanto este horizonte como el anterior se dan preferentemente en lugares bien iluminados. En lugares menos iluminados y posiblemente con la concurrencia de otros factores que se nos escapan, es notable el horizonte de Gelidium pussillum var. pulvinatum, formando un cesped denso por encima del nivel litoral como en Vilanova, o en la zona litoral: Gelidium crinale en Garraf, extendiendose la misma especie un poco por debajo. 
Zona infralitoral, nivel superior.

En lugares batidos, poco batidos y de iluminación variable es frecuente Corallina mediterranea que llega a formar densas poblaciones junto con otras especies (composición variable), prácticamente en todas las localidades estudiadas.

Facies de Petroglossum nicaeense observada en Pta del Canò (Sta Cristina) en lugar batido y con una orientación que reduce mucha su iluminación.

\section{Lugares encalmados}

Las poblaciones densas quedarán concretadas a la zona litoral, pues la supralitoral menos humedecida por las olas, sustentará ejemplares dispersos de diferentes especies; las poblaciones densas estarán en todo caso unos pocos centímetros por encima del nivel 0 metros.

\section{Zona litoral}

Poblaciones de Gelidium sp.pl., Laurencia obtusa, Falkenbergia rufolanosa como especies mas abundantes a las que acompañan otras que pueden presentar abundancia local: Padina pavonia, Colpomenia sinuosa, Corallina mediterranea, Jania rubens, etc., como en Pta Prima y El Molar en St. Feliu de Guíxols.

Poblaciones de Gelidium pussillum y Ulva lactuca acompañadas de Corallina mediterranea más o menos abundante.

Poblaciones de rocas enarenadas como las descritas en Garraf, con Enteromorpha linza, E. ramulosa, E. compressa, Ulva lactuca, U. rigida, Corallina, Gelidium y Gracilaria sp.

En realidad las poblaciones de lugares encalmados están relacionadas por lo general, o con sistemas litorales de rocas de escasa pendiente y cubetas, o bien con factores artificiales como son los puertos, donde a las condiciones de escaso movimiento debe añadirse la presencia de nutrientes en abundancia. Las cubetas pertenecen a la zona infralitoral y serán tratadas más adelante.

Facies de lugares encalmados y aguas contaminadas donde incluímos las poblaciones descritas en los puertos de Sant Feliu de Guíxols y Blanes aunque abarcan un poco por encima y por debajo de la zona litoral, con Bangia fuscopurpurea, Enteromorpha y Ulva lactuca, así como Scytosiphon lomentaria en Blanes, como principales especies. Codium tomentosum no es raro justo por debajo del nivel 0 metros.

Zona infralitoral, nivel superior.

Cubetas bien iluminadas con poblaciones de Padina pavonia, Halopteris scoparia y Cystoseira caespitosa entre otras.

Cubetas semiiluminadas como las de Garraf con Gymnogongrus norvegicus. 
Hendiduras con Dictyopteris membranacea y Taonia atomaria junto a Corallina mediterranea, como en Pta Prima en Sant Feliu de Guíxols.

En lugares más o menos batidos, el efecto del oleaje decrece con la profundidad de forma variable según las condiciones locales. En este caso el principal factor responsable de la presencia de unas comunidades u otras es la luz; diferenciamos entonces:

Poblaciones de Cladostephus verticillatus y Acetabularia mediterranea en lugares poco movidos a 2-3 m., de profundidad y bien iluminados, como en El Molar de Sant Feliu de Guíxols.

Poblaciones de Codium tomentosum y Codium bursa a 5-6 m., de profundidad en lugares iluminados a los que acompañan Dictyota dichotoma, Halopteris filicina y otras especies como en Sa Tuna, Begur.

En grietas y paredes verticales de escasa iluminación son comunes las poblaciones de Peyssonnelia sp. pl., Udotea petiolata y Halimeda tuna, que en algunos casos llegan hasta cerca del nivel 0 m., como en Pta Prima en St. Feliu de Guíxols.

\author{
BIBLIOGRAFIA
}

ARDRE, F. 1971. Contribution a l'etude des algues marines du Portugal. II, Ecologie et Chorologie. Bull. Cent. Etude. Rech. Sci., 8 (3): 359-574. Biarritz.

BELLAN-SANTINI, D. 1969. Contribution a l'etude des peuplements infralittoraux sur substrat rocheux (Etude qualitative et quantitative de la frange supérieur). Rec. Trav. St. mar. End. (63-47): 3-294.

BOUDOURESQUE, C. F. 1969. Etude quantitative et qualitative d'un peuplement algal a Cystoseira mediterranea dans la region de Banyuls-sur-Mer (P.O.). Vie et Milieu, 20 (2) ser. B: 437-452.

- 1970. Recherches sur les concepts de biocenose et de continum au niveau des peuplements benthiques sciaphiles. Vie et Milieu, 21 (1-B): 103-130.

EQUIPO DE BENTOS (varios autores). 1974. Estudio ecológico de las comunidades bentónicas de sustratos duros de la zona superior de la plataforma continental mediterránea española. Memoria final I y II. Inédito.

FELDMANN, J. 1937. Recherches sur la végétation marine de la Mediterranée. Rev. Algol., 10: 73-254.

FEOLI, E. \& BRESSAN, G. 1972. Affinità floristica dei tipi di vegetazione bentonica della Cala di Mitigliano. Giorn. Bot. Igal., 106: 245-256.

GIACCONE, G. \& LEO, A. 1967. Flora e vegetazione algale dei Golfo di Palermo. Lav. dell'Inst. di Bot. 22: 251.317. Palermo.

LEWIS, J.R. 1964. The ecology of Rocky Shores. London. 323 pp.

MOLINIER, R. 1960. Etude des biocénosis marines du Cap Corse. Vegetatio, Acta geobotanique, 9: I: 121-192; II: 217-312.

NIELL, X. 1976. Estudios sobre la estructura, dinámica y producción del fitobentos intermareal (facies rocosa) de la ría de Vigo. Tesis. Universidad de Barcelona.

PEREZ, J. M. \& PICART, J. 1964. Nouveau Manuel de Bionomie benthique de la mer Méditerranée. Rec. Trav. St. Mar. Endoume, 31 (47): 5-137.

PIGNATTI, S., CRISTINI, P. \& RIZZI, L. 1967. Le associazioni algali della Grota delle Viole nell'isola di S. Domino (Is. Tremiti). Giorn. Bot. Ital., 101: 117-126. 
POLO, L. \& SEOANE-CAMBA, J. 1976 a. Comunidades bentónicas de sustrato duro del litoral NE Español. XIII. Vegetación: Diversidad y Sociabilidad. Collect. Botanica (en prensa).

- \& SEOANE-CAMBA, J. 1976 b. Ibid., XVII. Distribución por profundidades de las especies de algas. Anal. Inst. Bot. Cavanilles (en prensa).

- 1978. Estudio sobre las algas bentónicas de la costa catalana. Tesis. Universidad Autónoma de Barcelona.

PRIETO, P. 1973. Sobre la vegetación del mar de Alborán. Cuad. C. Biol., 2 (1): 31-36.

SEOANE-CAMBA, J. 1965. Estudios sobre las algas bentónicas de la costa sur de la Península Ibérica (Litoral de Cádiz). Inv. Pesq., 29: 3-216.

- 1969. Sobre la zonación del sistema litoral y su nomenclatura. Inv. Pesq., 33 (1): 261267.

- 1975. Algas bentónicas españolas en los Herbarios Thuret-Bornet y Sauvageau del Museum National d'Histoire Naturelle de París. II. Algas de Cataluña y Baleares (excepto Menorca). Anal. Inst. Bot. Cavanilles, 32 (2): 33-51.

- \& POLO, L. 1974. Estudio botánico de la Plataforma submarina catalana. Anal. Inst. Bot. Cavanilles, 31: 179-183.

- \& POLO, 1977. Comunidades bentónicas de sustrato duro del litoral NE Español. XIX. Vegetación: Sistemática, Fenología y novedades para la flora española. Anal. Inst. Bot. Cavanilles (en prensa). 